\title{
Cyclic sieving for longest reduced words in the hyperoctahedral group
}

\author{
T. K. Petersen ${ }^{1}$ and L. Serrano" $\|^{t}$ \\ ${ }^{1}$ DePaul University, Chicago, IL \\ ${ }^{2}$ University of Michigan, Ann Arbor, $M I$
}

received $21^{\text {st }}$ July 2010 ,

\begin{abstract}
We show that the set $R\left(w_{0}\right)$ of reduced expressions for the longest element in the hyperoctahedral group exhibits the cyclic sieving phenomenon. More specifically, $R\left(w_{0}\right)$ possesses a natural cyclic action given by moving the first letter of a word to the end, and we show that the orbit structure of this action is encoded by the generating function for the major index on $R\left(w_{0}\right)$.

Résumé. Nous montrons que l'ensemble $R\left(w_{0}\right)$ des expressions réduites pour l'élément le plus long du groupe hyperoctaédral présente le phénomène cyclique de tamisage. Plus précisément, $R\left(w_{0}\right)$ possède une action naturelle cyclique donnée par le déplacement de la première lettre d'un mot vers la fin, et nous montrons que la structure d'orbite de cette action est codée par la fonction génératrice pour l'indice majeur sur $R\left(w_{0}\right)$.

Resumen. En este artículo demostramos que el conjunto $R\left(w_{0}\right)$ de expresiones reducidas del elemento mas largo del grupo hiperoctaedro presenta el fenómeno de tamizado cíclico. Para ser mas precisos, $R\left(w_{0}\right)$ posee una acción cíclica natural dada por el movimiento de la primera letra de una palabra al final, y nosotros mostramos que la estructura de las orbitas de esta acción está codificada por la función generatriz del indice mayor en $R\left(w_{0}\right)$.
\end{abstract}

Keywords: Cyclic sieving, hyperoctahedral group, standard Young tableau, shifted staircase, reduced word

\section{Introduction and main result}

Suppose we are given a finite set $X$, a finite cyclic group $C=\langle\omega\rangle$ acting on $X$, and a polynomial $X(q) \in \mathbb{Z}[q]$ with integer coefficients. Following Reiner, Stanton, and White [RSW], we say that the triple $(X, C, X(q))$ exhibits the cyclic sieving phenomenon (CSP) if for every integer $d \geq 0$, we have that $\left|X^{\omega^{d}}\right|=X\left(\zeta^{d}\right)$ where $\zeta \in \mathbb{C}$ is a root of unity of multiplicitive order $|C|$ and $X^{\omega^{d}}$ is the fixed point set of the action of the power $\omega^{d}$. In particular, since the identity element fixes everything in any group action, we have that $|X|=X(1)$ whenever $(X, C, X(q))$ exhibits the CSP.

If the triple $(X, C, X(q))$ exhibits the CSP and $\zeta$ is a primitive $|C|^{\text {th }}$ root of unity, we can determine the cardinalities of the fixed point sets $X^{1}=X, X^{\omega}, X^{\omega^{2}}, \ldots, X^{\omega^{|C|-1}}$ via the polynomial evaluations $X(1), X(\zeta), X\left(\zeta^{2}\right), \ldots, X\left(\zeta^{|C|-1}\right)$. These fixed point set sizes determine the cycle structure of the

\footnotetext{
$\dagger$ Partially supported by NSF grant DMS-0555880

1365-8050 (c) 2010 Discrete Mathematics and Theoretical Computer Science (DMTCS), Nancy, France
} 
canonical image of $\omega$ in the group of permutations of $X, S_{X}$. Therefore, to find the cycle structure of the image of any bijection $\omega: X \rightarrow X$, it is enough to determine the order of the action of $\omega$ on $X$ and find a polynomial $X(q)$ such that $(X,\langle c\rangle, X(q))$ exhibits the CSP.

The cyclic sieving phenomenon has been demonstrated in a variety of contexts. The paper of Reiner, Stanton, and White [RSW] itself includes examples involving noncrossing partitions, triangulations of polygons, and cosets of parabolic subgroups of Coxeter groups. An example of the CSP with standard Young tableaux is due to Rhoades [Rh] and will discussed further in Section 4 Now we turn to the CSP of interest to this note.

Let $w_{0}=w_{0}^{\left(B_{n}\right)}$ denote the longest element in the type $B_{n}$ Coxeter group. Given generating set $S=\left\{s_{1}, \ldots, s_{n}\right\}$ for $B_{n},\left(s_{1}\right.$ being the "special" reflection), we will write a reduced expression for $w_{0}$ as a word in the subscripts. For example, $w_{0}^{\left(B_{3}\right)}$ can be written as

$$
s_{1} s_{2} s_{1} s_{3} s_{2} s_{3} s_{1} s_{2} s_{3}
$$

we will abbreviate this product by 121323123 . It turns out that if we cyclically permute these letters, we always get another reduced expression for $w_{0}$. Said another way, $s_{i} w_{0} s_{i}=w_{0}$ for $i=1, \ldots, n$. The same is not true for longest elements of other classical types. In type A, we have $s_{i} w_{0}^{\left(A_{n}\right)} s_{n+1-i}=w_{0}^{\left(A_{n}\right)}$, and for type $\mathrm{D}$,

$$
w_{0}^{\left(D_{n}\right)}= \begin{cases}s_{i} w_{0}^{\left(D_{n}\right)} s_{i} & \text { if } n \text { even or } i>2, \\ s_{i} w_{0}^{\left(D_{n}\right)} s_{3-i} & \text { if } n \text { odd and } i=1,2 .\end{cases}
$$

Let $R\left(w_{0}\right)$ denote the set of reduced expressions for $w_{0}$ in type $B_{n}$ and let $c: R\left(w_{0}\right) \rightarrow R\left(w_{0}\right)$ denote the action of placing the first letter of a word at the end. Then the orbit in $R\left(w_{0}^{\left(B_{3}\right)}\right)$ of the word above is:

$$
\begin{gathered}
\{121323123 \rightarrow 213231231 \rightarrow 132312312 \rightarrow 323123121 \rightarrow 231231213 \\
\rightarrow 312312132 \rightarrow 123121323 \rightarrow 231213231 \rightarrow 312132312\} .
\end{gathered}
$$

As the length of $w_{0}$ is $n^{2}$, we clearly have $c^{n^{2}}=1$, and the size of any orbit divides $n^{2}$. For an example of a smaller orbit, notice that the word 213213213 has cyclic order 3.

For any word $w=w_{1} \ldots w_{l}$, (e.g., a reduced expression for $w_{0}$ ), a descent of $w$ is defined to be a position $i$ in which $w_{i}>w_{i+1}$. The major index of $w, \operatorname{maj}(w)$, is defined as the sum of the descent positions. For example, the word $w=121323123$ has descents in positions 2,4 , and 6 , so its major index is $\operatorname{maj}(w)=2+4+6=12$. Let $f_{n}(q)$ denote the generating function for this statistic on words in $R\left(w_{0}\right)$ :

$$
f_{n}(q)=\sum_{w \in R\left(w_{0}\right)} q^{\operatorname{maj}(w)} .
$$

The following is our main result.

Theorem 1 The triple $\left(R\left(w_{0}\right),\langle c\rangle, X(q)\right)$ exhibits the cyclic sieving phenomenon, where

$$
X(q)=q^{-n\left(\begin{array}{l}
n \\
2
\end{array}\right)} f_{n}(q) .
$$


For example, let us consider the case $n=3$. We have

$$
\begin{aligned}
X(q)= & q^{-9} \sum_{w \in R\left(w_{0}^{\left(B_{3}\right)}\right)} q^{\operatorname{maj}(w)} \\
= & 1+q^{2}+2 q^{3}+2 q^{4}+2 q^{5}+4 q^{6}+3 q^{7}+4 q^{8}+4 q^{9} \\
& +4 q^{10}+3 q^{11}+4 q^{12}+2 q^{13}+2 q^{14}+2 q^{15}+q^{16}+q^{18} .
\end{aligned}
$$

Let $\zeta=e^{\frac{2 \pi i}{9}}$. Then we compute:

$$
\begin{array}{lll}
X(1)=42 & X\left(\zeta^{3}\right)=6 & X\left(\zeta^{6}\right)=6 \\
X(\zeta)=0 & X\left(\zeta^{4}\right)=0 & X\left(\zeta^{7}\right)=0 \\
X\left(\zeta^{2}\right)=0 & X\left(\zeta^{5}\right)=0 & X\left(\zeta^{8}\right)=0
\end{array}
$$

Thus, the 42 reduced expressions for $w_{0}^{\left(B_{3}\right)}$ split into two orbits of size three (the orbits of 123123123 and 132132132) and four orbits of size nine.

We prove Theorem 1 by relating it to another instance of the CSP, namely Rhoades' recent (and deep) result [Rh, Thm 3.9] for the set $S Y T\left(n^{m}\right)$ of rectangular standard Young tableaux with respect to the action of promotion (defined in Section 2). To make the connection, we rely on a pair of remarkable bijections due to Haiman [H1, [H2]. The composition of Haiman's bijections maps to $R\left(w_{0}\right)$ from the set of square tableaux, $S Y T\left(n^{n}\right)$. In this note our main goal is to show that Haiman's bijections carry the orbit structure of promotion on $S Y T\left(n^{n}\right)$ to the orbit structure of $c$ on $R\left(w_{0}\right)$.

We conclude this section by remarking that this approach was first outlined by Rhoades [Rh, Thm 8.1]. One purpose of this article is to fill some nontrivial gaps in his argument. A second is to justify the new observation that the polynomial $X(q)$ can be expressed as the generating function for the major index on $R\left(w_{0}\right)$.

\section{Promotion on standard Young tableaux}

For $\lambda$ a partition, let $S Y T(\lambda)$ denote the set of standard Young tableaux of shape $\lambda$. If $\lambda$ is a strict partition, i.e., with no equal parts, then let $S Y T^{\prime}(\lambda)$ denote the set of standard Young tableaux of shifted shape $\lambda$. We now describe the action of jeu de taquin promotion, first defined by Schützenberger [Sch].

We will consider promotion as a permutation of tableaux of a fixed shape (resp. shifted shape), $p$ : $S Y T(\lambda) \rightarrow S Y T(\lambda)$ (resp. $p: S Y T^{\prime}(\lambda) \rightarrow S Y T^{\prime}(\lambda)$ ). Given a tableau $T$ with $\lambda \vdash n$, we form $p(T)$ with the following algorithm. (We denote the entry in row $a$, column $b$ of a tableau $T$, by $T_{a, b}$.)

1. Remove the entry 1 in the upper left corner and decrease every other entry by 1 . The empty box is initialized in position $(a, b)=(1,1)$.

2. Perform jeu de taquin:

(a) If there is no box to the right of the empty box and no box below the empty box, then go to 3).

(b) If there is a box to the right or below the empty box, then swap the empty box with the box containing the smaller entry, i.e., $p(T)_{a, b}:=\min \left\{T_{a, b+1}, T_{a+1, b}\right\}$. Set $(a, b):=\left(a^{\prime}, b^{\prime}\right)$, where $\left(a^{\prime}, b^{\prime}\right)$ are the coordinates of box swapped, and go to $\left.2 a\right)$. 
3. Fill the empty box with $n$.

Here is an example:

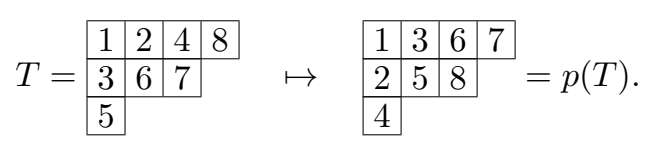

As a permutation, promotion naturally splits $S Y T(\lambda)$ into disjoint orbits. For a general shape $\lambda$ there seems to be no obvious pattern to the sizes of the orbits. However, for certain shapes, notably Haiman's "generalized staircases" more can be said [H2] (see also Edelman and Greene [EG, Cor. 7.23]). In particular, rectangles fall into this category, with the following result.

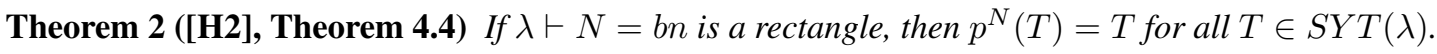

Thus for $n \times n$ square shapes $\lambda, p^{n^{2}}=1$ and the size of every orbit divides $n^{2}$. With $n=3$, here is an orbit of size 3 :

$$
\begin{array}{|l|l|l}
\hline 1 & 2 & 5 \\
\hline 3 & 6 & 8 \\
\hline 4 & 7 & 9 \\
\hline
\end{array} \rightarrow \rightarrow \begin{array}{|l|l|l|}
\hline 1 & 4 & 7 \\
\hline 2 & 5 & 8 \\
\hline 3 & 6 & 9 \\
\hline
\end{array} \rightarrow \rightarrow \begin{array}{|l|l|l|}
\hline 1 & 3 & 6 \\
\hline 2 & 4 & 7 \\
\hline 5 & 8 & 9 \\
\hline
\end{array} \rightarrow \cdots
$$

There are 42 standard Young tableaux of shape $(3,3,3)$, and there are 42 reduced expressions in the set $R\left(w_{0}^{\left(B_{3}\right)}\right)$. Stanley first conjectured that $R\left(w_{0}^{\left(B_{3}\right)}\right)$ and $S Y T\left(n^{n}\right)$ are equinumerous, and Proctor suggested that rather than $S Y T\left(n^{n}\right)$, a more direct correspondence might be given with $S Y T^{\prime}(2 n-$ $1,2 n-3, \ldots, 1)$, that is, with shifted standard tableaux of "doubled staircase" shape. (That the squares and doubled staircases are equinumerous follows easily from hook length formulas.)

Haiman answers Proctor's conjecture in such a way that the structure of promotion on doubled staircases corresponds precisely to cyclic permutation of words in $R\left(w_{0}\right)$ [ [H2, Theorem 5.12]. Moreover, in [H1, Proposition 8.11], he gives a bijection between standard Young tableaux of square shape and those of doubled staircase shape that (as we will show) commutes with promotion.

As an example, his bijection carries the orbit in 1 to this shifted orbit:

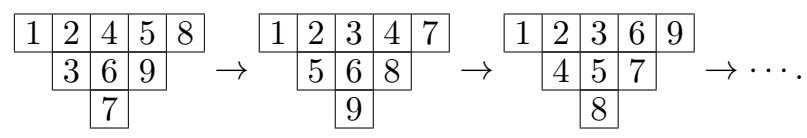

Both of these orbits of tableaux correspond to the orbit of the reduced word 132132132.

\section{Haiman's bijections}

We first describe the bijection between reduced expressions and shifted standard tableaux of doubled staircase shape. This bijection is described in Section 5 of [H2].

Let $T$ in $S Y T^{\prime}(2 n-1,2 n-3, \ldots, 1)$. Notice the largest entry in $T$, (i.e., $\left.n^{2}\right)$, occupies one of the outer corners. Let $r(T)$ denote the row containing this largest entry, numbering the rows from the bottom up. The promotion sequence of $T$ is defined to be $\Phi(T)=r_{1} \cdots r_{n^{2}}$, where $r_{i}=r\left(p^{i}(T)\right)$. Using the example above of

$$
T=\begin{array}{|l|l|l|l|l|}
\hline 1 & 2 & 4 & 5 & 8 \\
\hline & 3 & 6 & 9 & \\
\cline { 2 - 3 } & 7 & \multicolumn{1}{|l}{}
\end{array}
$$


we see $r(T)=2, r(p(T))=1, r\left(p^{2}(T)\right)=3$, and since $p^{3}(T)=T$, we have

$$
\Phi(T)=132132132 .
$$

Haiman's result is the following.

Theorem 3 ([[H2], Theorem 5.12) The map $T \mapsto \Phi(T)$ is a bijection $S Y T^{\prime}(2 n-1,2 n-3, \ldots, 1) \rightarrow$ $R\left(w_{0}\right)$.

By construction, one can see that applying promotion to $T$ will cyclically shift the letters in $\Phi(T)$. Therefore, we have

$$
\Phi(p(T))=c(\Phi(T))
$$

i.e., $\Phi$ is an orbit-preserving bijection

$$
\left(S Y T^{\prime}(2 n-1,2 n-3, \ldots, 1), p\right) \longleftrightarrow\left(R\left(w_{0}\right), c\right) .
$$

Next, we will describe the bijection

$$
H: S Y T\left(n^{n}\right) \rightarrow S Y T^{\prime}(2 n-1,2 n-3, \ldots, 1)
$$

between squares and doubled staircases. Though not obvious from the definition below, we will demonstrate that $H$ commutes with promotion.

We assume the reader is familiar with the Robinson-Schensted-Knuth insertion algorithm (RSK). (See [Sta, Section 7.11], for example.) This is a map between words $w$ and pairs of tableaux $(P, Q)=$ $(P(w), Q(w))$. We say $P$ is the insertion tableau and $Q$ is the recording tableau.

There is a similar correspondence between words $w$ and pairs of shifted tableaux $\left(P^{\prime}, Q^{\prime}\right)=\left(P^{\prime}(w), Q^{\prime}(w)\right)$ called shifted mixed insertion due to Haiman $[\overline{\mathrm{H} 1}]$. (See also Sagan [Sa] and Worley [W].) Serrano defined a semistandard generalization of shifted mixed insertion in [Ser]. Throughout this paper we refer to semistandard shifted mixed insertion simply as mixed insertion. Details can be found in [Ser, Section $1.1]$.

Theorem 4 ([Ser] Theorem 2.26) Let $w$ be a word. If we view $Q(w)$ as a skew shifted standard Young tableau and apply jeu de taquin to obtain a standard shifted Young tableau, the result is $Q^{\prime}(w)$ (independent of any choices in applying jeu de taquin).

For example, if $w=332132121$, then

$$
\begin{aligned}
& (P, Q)=\left(\begin{array}{|l|l|l|l|l|l|}
\hline 1 & 1 & 1 \\
\hline 2 & 2 & 2 \\
\hline 3 & 3 & 3 & 1 & 2 & 5 \\
\hline 3 & 6 & 8 \\
\hline 4 & 7 & 9 \\
\hline
\end{array}\right),
\end{aligned}
$$

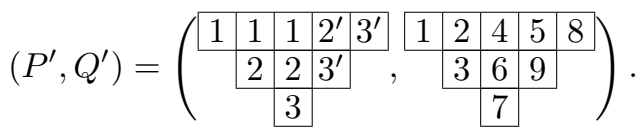

Performing jeu de taquin we see:

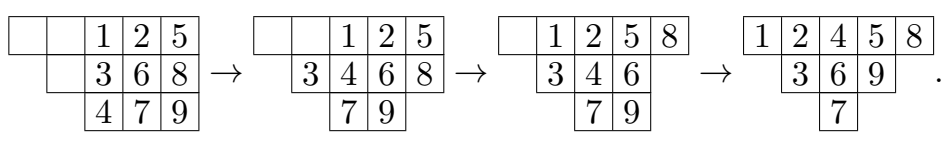


Haiman's bijection is precisely $H(Q)=Q^{\prime}$. That is, given a standard square tableau $Q$, we embed it in a shifted shape and apply jeu de taquin to create a standard shifted tableau. That this is indeed a bijection follows from Theorem 4, but is originally found in [H1, Proposition 8.11].

Remark 5 Haiman's bijection $H$ applies more generally between rectangles and "shifted trapezoids", i.e., for $m \leq n$, we have $H: S Y T\left(n^{m}\right) \rightarrow S Y T^{\prime}(n+m-1, n+m-3, \ldots, n-m+1)$. All the results presented here extend to this generality, with similar proofs. We restict to squares and doubled staircases for clarity of exposition.

We will now fix the tableaux $P$ and $P^{\prime}$ to ensure that the insertion word $w$ has particularly nice properties. We will use the following lemma.

Lemma 6 ([|Ser], Proposition 1.8) Fix a word $w$. Let $P=P(w)$ be the RSK insertion tableau and let $P^{\prime}=P^{\prime}(w)$ be the mixed insertion tableau. Then the set of words that mixed insert into $P^{\prime}$ is contained in the set of words that RSK insert into $P$.

Now we apply Lemma 6 to the word

$$
w=\underbrace{n \cdots n}_{n} \cdots \underbrace{2 \cdots 2}_{n} \underbrace{1 \cdots 1}_{n}
$$

If we use RSK insertion, we find $P$ is an $n \times n$ square tableau with all $1 \mathrm{~s}$ in row first row, all $2 \mathrm{~s}$ in the second row, and so on. With such a choice of $P$ it is not difficult to show that any other word $u$ inserting to $P$ has the property that in any initial subword $u_{1} \cdots u_{i}$, there are at least as many letters $(j+1)$ as letters $j$. Such words are sometimes called (reverse) lattice words or (reverse) Yamanouchi words. Notice also that any such $u$ has $n$ copies of each letter $i, i=1, \ldots, n$. We call the words inserting to this choice of $P$ square words.

On the other hand, if we use mixed insertion on $w$, we find $P^{\prime}$ as follows (with $n=4$ ):

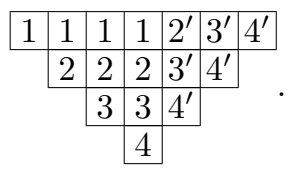

In general, on the "shifted half" of the tableau we see all $1 \mathrm{~s}$ in the first row, all $2 \mathrm{~s}$ in the second row, and so on. In the "straight half" we see only prime numbers, with $2^{\prime}$ on the first diagonal, $3^{\prime}$ on the second diagonal, and so on. Lemma 6 tells us that every $u$ that mixed inserts to $P^{\prime}$ is a square word. But since the sets of recording tableaux for $P$ and for $P^{\prime}$ are equinumerous, we see that the set of words mixed inserting to $P^{\prime}$ is precisely the set of all square words.

Remark 7 Yamanouchi words give a bijection with square standard Young tableaux that circumvents insertion completely. In reading the word from left to right, if $w_{i}=j$, we put letter $i$ in the leftmost unoccupied position of row $n+1-j$. (See [Sta. Proposition 7.10.3(d)].)

We will soon characterize promotion in terms of operators on insertion words. First, some lemmas.

For a tableau $T$ (shifted or not) let $\Delta T$ denote the result of all but step (3) of promotion. That is, we delete the smallest entry and perform jeu de taquin, but we do not fill in the empty box. The following lemma says that, in both the shifted and unshifted cases, this can be expressed very simply in terms of our insertion word. The first part of the lemma is a direct application of the theory of jeu de taquin (see, e.g., [Sta A1.2]); the second part is [Ser, Lemma 3.9]. 
Lemma 8 For a word $w=w_{1} w_{2} \cdots w_{l}$, let $\widehat{w}=w_{2} \cdots w_{l}$. Then we have

$$
Q(\widehat{w})=\Delta Q(w),
$$

and

$$
Q^{\prime}(\widehat{w})=\Delta Q^{\prime}(w)
$$

The operator $e_{j}$ acting on words $w=w_{1} \cdots w_{l}$ is defined in the following way. Consider the subword of $w$ formed only by the letters $j$ and $j+1$. Consider every $j+1$ as an opening bracket and every $j$ as a closing bracket, and pair them up accordingly. The remaining word is of the form $j^{r}(j+1)^{s}$. The operator $e_{j}$ leaves all of $w$ invariant, except for this subword, which it changes to $j^{r-1}(j+1)^{s+1}$ (assuming $r>0$, otherwise $e_{j}$ is not defined on $w$ ). This operator is widely used in the theory of crystal graphs.

As an example, we calculate $e_{2}(w)$ for the word $w=3121221332$. The subword formed from the letters 3 and 2 is

$$
3 \cdot 2 \cdot 22 \cdot 332
$$

which corresponds to the bracket sequence ()$))(()$. Removing paired brackets, one obtains $))($, corresponding to the subword

$$
\cdots 22 \cdot 3 \cdots
$$

We change the last 2 to a 3 and keep the rest of the word unchanged, obtaining $e_{2}(w)=3121231332$.

The following lemma shows that this operator leaves the recording tableau unchanged. The unshifted case is found in work of Lascoux, Leclerc, and Thibon [LLT, Theorem 5.5.1]; the shifted case follows from the unshifted case, and the fact that the mixed recording tableau of a word is uniquely determined by its RSK recording tableau (Theorem 4 ).

Lemma 9 Recording tableaux are invariant under the operators $e_{i}$. That is,

$$
Q\left(e_{i}(w)\right)=Q(w)
$$

and

$$
Q^{\prime}\left(e_{i}(w)\right)=Q^{\prime}(w)
$$

Let $\bar{e}=e_{1} \cdots e_{n-1}$ denote the composite operator given by applying first $e_{n-1}$, then $e_{n-2}$ and so on. It is clear that if $w=w_{1} \cdots w_{n^{2}}$ is a square word, then $\bar{e}(\widehat{w}) 1$ is again a square word.

Theorem 10 Let $w=w_{1} \cdots w_{n^{2}}$ be a square word. Then,

$$
p(Q(w))=Q(\bar{e}(\widehat{w}) 1),
$$

and

$$
p\left(Q^{\prime}(w)\right)=Q^{\prime}(\bar{e}(\widehat{w}) 1) .
$$

In other words, Haiman's bijection commutes with promotion:

$$
p(H(Q))=H(p(Q)) .
$$


Proof: By Lemma 8 we see that $Q(\widehat{w})$ is only one box away from $p(Q(w))$. Further, repeated application of Lemma 9 shows that

$$
Q(\widehat{w})=Q\left(e_{n-1}(\widehat{w})\right)=Q\left(e_{n-2}\left(e_{n-1}(\widehat{w})\right)\right)=\cdots=Q(\bar{e}(\widehat{w})) .
$$

The same lemmas apply show $Q^{\prime}(\bar{e}(\widehat{w}))$ is one box away from $p\left(Q^{\prime}(w)\right)$.

All that remains is to check that the box added by inserting 1 into $P(\bar{e}(\widehat{w}))$ (resp. $P^{\prime}(\bar{e}(\widehat{w}))$ ) is in the correct position. But this follows from the observation that $\bar{e}(\widehat{w}) 1$ is a square word, and square words insert (resp. mixed insert) to squares (resp. doubled staircases).

\section{Rhoades' result}

Rhoades [Rh] proved an instance of the CSP related to the action of promotion on rectangular tableaux. His result is quite deep, employing Kahzdan-Lusztig cellular representation theory in its proof.

Recall that for any partition $\lambda \vdash n$, we have that the standard tableaux of shape $\lambda$ are enumerated by the Frame-Robinson-Thrall hook length formula:

$$
f^{\lambda}=|S Y T(\lambda)|=\frac{n !}{\prod_{(i, j) \in \lambda} h_{i j}},
$$

where the product is over the boxes $(i, j)$ in $\lambda$ and $h_{i j}$ is the hook length at the box $(i, j)$, i.e., the number of boxes directly east or south of the box $(i, j)$ in $\lambda$, counting itself exactly once. To obtain the polynomial used for cyclic sieving, we replace the hook length formula with a natural $q$-analogue. First, recall that for any $n \in \mathbb{N},[n]_{q}:=1+q+\cdots+q^{n-1}$ and $[n]_{q} !:=[n]_{q}[n-1]_{q} \cdots[1]_{q}$.

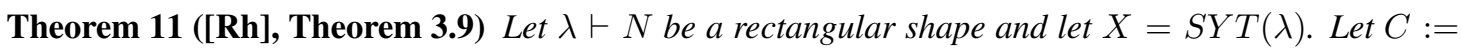
$\mathbb{Z} / N \mathbb{Z}$ act on $X$ via promotion. Then, the triple $(X, C, X(q))$ exhibits the cyclic sieving phenomenon, where

$$
X(q)=\frac{[N]_{q} !}{\Pi_{(i, j) \in \lambda}\left[h_{i j}\right]_{q}}
$$

is the q-analogue of the hook length formula.

Now thanks to Theorem 10 we know that $H$ preserves orbits of promotion, and as a consequence we see the CSP for doubled staircases.

Corollary 12 Let $X=S Y T^{\prime}(2 n-1,2 n-3, \ldots, 1)$, and let $C:=\mathbb{Z} / n^{2} \mathbb{Z}$ act on $X$ via promotion. Then the triple $(X, C, X(q))$ exhibits the cyclic sieving phenomenon, where

$$
X(q)=\frac{\left[n^{2}\right]_{q} !}{[n]_{q}^{n} \prod_{i=1}^{n-1}\left([i]_{q} \cdot[2 n-i]_{q}\right)^{i}}
$$

is the q-analogue of the hook length formula for an $n \times n$ square Young diagram.

Because of Theorem 3 the set $R\left(w_{0}\right)$ also exhibits the CSP.


act on $X$ by cyclic rotation of words. Then the triple $(X, C, X(q))$ exhibits the cyclic sieving phenomenon. 
Corollary 13 is the CSP for $R\left(w_{0}\right)$ as stated by Rhoades. This is nearly our main result (Theorem 1 , but for the definition of $X(q)$.

In spirit, if $(X, C, X(q))$ exhibits the CSP, the polynomial $X(q)$ should be some $q$-enumerator for the set $X$. That is, it should be expressible as

$$
X(q)=\sum_{x \in X} q^{s(x)}
$$

where $s$ is an intrinsically defined statistic for the elements of $X$. Indeed, nearly all known instances of the cyclic sieving phenomenon have this property. For example, it is known ([Sta, Cor 7.21.5]) that the $q$-analogue of the hook-length formula can be expressed as follows:

$$
f^{\lambda}(q)=q^{-\kappa(\lambda)} \sum_{T \in S Y T(\lambda)} q^{\operatorname{maj}(T)},
$$

where $\kappa\left(\lambda_{1}, \ldots, \lambda_{l}\right)=\sum_{1 \leq i \leq l}(i-1) \lambda_{i}$ and for a tableau $T, \operatorname{maj}(T)$ is the sum of all $i$ such that $i$ appears in a row above $i+1$. Thus $X(q)$ in Theorem 11 can be described in terms of a statistic on Young tableaux.

With this point of view, Corollaries 12 and 13 are aesthetically unsatisfying. Section 5 is given to showing that $X(q)$ can be defined as the generating function for the major index on words in $R\left(w_{0}\right)$. It would be interesting to find a combinatorial description for $X(q)$ in terms of a statistic on $S Y T^{\prime}(2 n-$ $1,2 n-3, \ldots, 1)$ as well, though we have no such description at present.

\section{Combinatorial description of $X(q)$}

As stated in the introduction, we will show that

$$
X(q)=q^{-n\left(\begin{array}{c}
n \\
2
\end{array}\right)} \sum_{w \in R\left(w_{0}\right)} q^{\operatorname{maj}(w)} .
$$

If we specialize equation 2 to square shapes, we see that $\kappa\left(n^{n}\right)=n\left(\begin{array}{c}n \\ 2\end{array}\right)$ and

$$
X(q)=q^{-n\left(\begin{array}{l}
n \\
2
\end{array}\right)} \sum_{T \in S Y T\left(n^{n}\right)} q^{\operatorname{maj}(T)} .
$$

Thus it suffices to exhibit a bijection between square tableaux and words in $R\left(w_{0}\right)$ that preserves major index. In fact, the composition $\Psi:=\Phi H$ has a stronger feature.

Define the cyclic descent set of a word $w=w_{1} \cdots w_{l}$ to be the set

$$
D(w)=\left\{i: w_{i}>w_{i+1}\right\} \quad(\bmod l)
$$

That is, we have descents in the usual way, but also a descent in position 0 if $w_{l}>w_{1}$. Then maj $(w)=$ $\sum_{i \in D(w)} i$. For example with $w=132132132, D(w)=\{0,2,3,5,6,8\}$ and maj $(w)=0+2+3+5+$ $6+8=24$.

Similarly, we follow $[\overline{\mathrm{Rh}}]$ in defining the cyclic descent set of a square (in general, rectangular) Young tableau. For $T$ in $S Y T\left(n^{n}\right)$, define $D(T)$ to be the set of all $i$ such that $i$ appears in a row above $i+1$, 
along with 0 if $n^{2}-1$ is above $n^{2}$ in $p(T)$. Major index is $\operatorname{maj}(T)=\sum_{i \in D(T)} i$. We will see that $\Psi$ preserves cyclic descent sets, and hence, major index. Using our earlier example of $w=132132132$, one can check that

$$
T=\Psi^{-1}(w)=\begin{array}{|l|l|l|}
\hline 1 & 2 & 5 \\
\hline 3 & 6 & 8 \\
\hline 4 & 7 & 9 \\
\hline
\end{array}
$$

has $D(T)=D(w)$, and so $\operatorname{maj}(T)=\operatorname{maj}(w)$.

Lemma 14 Let $T \in S Y T\left(n^{n}\right)$, and let $w=\Psi(T)$ in $R\left(w_{0}\right)$. Then $D(T)=D(w)$.

Proof: First, we observe that both types of descent sets shift cyclically under their respective actions:

$$
D(p(T))=\left\{i-1 \quad\left(\bmod n^{2}\right): i \in D(T)\right\},
$$

and

$$
D(c(w))=\left\{i-1 \quad\left(\bmod n^{2}\right): i \in D(w)\right\} .
$$

For words under cyclic rotation, this is obvious. For tableaux under promotion, this is a lemma of Rhoades [Rh, Lemma 3.3].

Because of this cyclic shifting, we see that $i \in D(T)$ if and only if $0 \in D\left(p^{i}(T)\right)$. Thus, it suffices to show that $0 \in D(T)$ if and only if $0 \in D(w)$. (Actually, it is easier to determine if $n^{2}-1$ is a descent.)

Let $S=\Phi^{-1}(w)$ be the shifted doubled staircase tableau corresponding to $w$. We have $n^{2}-1 \in D(w)$ if and only if $n^{2}$ is in a higher row in $p^{-1}(S)$ than in $S$. But since $n^{2}$ occupies the same place in $p^{-1}(S)$ as $n^{2}-1$ occupies in $S$, this is to say $n^{2}-1$ is above $n^{2}$ in $S$. On the other hand, $n^{2}-1 \in D(T)$ if and only if $n^{2}-1$ is above $n^{2}$ in $T$. It is straightforward to check that since $S$ is obtained from $T$ by jeu de taquin into the upper corner, the relative heights of $n^{2}$ and $n^{2}-1$ (i.e., whether $n^{2}$ is below or not) are the same in $S$ as in $T$. This completes the proof.

This lemma yields the desired result for $X(q)$.

Theorem 15 The q-analogue of the hook length formula for an $n \times n$ square Young diagram is, up to a shift, the major index generating function for reduced expressions of the longest element in the hyperoctahedral group:

$$
\sum_{w \in R\left(w_{0}\right)} q^{\operatorname{maj}(w)}=q^{n\left(\begin{array}{c}
n \\
2
\end{array}\right)} \cdot \frac{\left[n^{2}\right]_{q} !}{[n]_{q}^{n} \prod_{i=1}^{n-1}\left([i]_{q} \cdot[2 n-i]_{q}\right)^{i}} .
$$

Theorem 15, along with Corollary 13, completes the proof of our main result, Theorem 1 . Because this result can be stated purely in terms of the set $R\left(w_{0}\right)$ and a natural statistic on this set, it would be interesting to obtain a self-contained proof, i.e., one that does not appeal to Haiman's or Rhoades' work. Why must a result about cyclic rotation of words rely on promotion of Young tableaux?

\section{Acknowledgements}

We thank Brendon Rhoades for encouraging us to write this note. Thanks also to Kevin Dilks, John Stembridge, and Alex Yong for fruitful discussions on this and related topics, and to Sergey Fomin for comments on the manuscript. 


\section{References}

[EG] P. Edelman and C. Greene: Balanced tableaux, Adv. in Math. 63 (1987), 42-99.

[H1] M. HAIMAN: On mixed insertion, symmetry, and shifted Young tableaux, J. Combin. Theory Ser. A 50 (1989), no. 2, 196-225.

[H2] M. HAIMAN: Dual equivalence with applications, including a conjecture of Proctor, Discrete Math. 99 (1992), 79-113.

[LlT] A. Lascoux, B. Leclerc, And J. -Y. Thibon: The plactic monoid, in "M. Lothaire, Algebraic combinatorics on words", Cambridge University Press, Cambridge, 2002 (Chapter 6).

[RSW] V. Reiner, D. Stanton, And D. White: The cyclic sieving phenomenon, J. Combin. Theory Ser. A 108 (2004), no. 1, 17-50.

[Rh] B. RhOADES: Cyclic sieving, promotion, and representation theory, J. Combin. Theory Ser. A 117 (2010), no. 1, 38-76.

[Sa] B. SAGAN: Shifted tableaux, Schur $Q$-functions, and a conjecture of R. P. Stanley, J. Combin. Theory Ser. A 45 (1987), 62-103.

[Ser] L. SERRANO: The shifted plactic monoid, arXiv: 0811.2057.

[Sch] M. P. SchütZenberger: Promotion des morphismes d'ensembles ordonnés, Discrete Mathematics 2, (1972), 73-94.

[Sta] R. StanleY: Enumerative Combinatorics Vol 2, Cambridge University Press, Cambridge, UK, 1999.

[W] D. R. WORLEY: A theory of shifted Young tableaux, Ph.D. thesis, MIT, 1984; available at http://hdl.handle.net/1721.1/15599. 
